\title{
Polypharmacology of small molecules targeting the ubiquitin- proteasome and ubiquitin-like systems
}

\author{
Ivano Amelio ${ }^{1}$, Vivien Landré ${ }^{1}$, Richard A. Knight ${ }^{1}$, Andrey Lisitsa ${ }^{2}$, Gerry Melino ${ }^{1,3}$ \\ and Alexey V. Antonov ${ }^{1}$ \\ ${ }^{1}$ Medical Research Council Toxicology Unit, Leicester, UK \\ 2 Institute of Biomedical Chemistry of The Russian Academy of Medical Sciences, Moscow, Russia \\ ${ }^{3}$ Department of Experimental Medicine \& Surgery, University of Rome "Tor Vergata", Rome, Italy \\ Correspondence to: Alexey V. Antonov, email: aa668@le.ac.uk
}

Keywords: UPS, UBL, SUMO, UBC13, UCH37

Received: March 09, $2015 \quad$ Accepted: April 03, $2015 \quad$ Published: April 23, 2015

This is an open-access article distributed under the terms of the Creative Commons Attribution License, which permits unrestricted use, distribution, and reproduction in any medium, provided the original author and source are credited.

\section{ABSTRACT}

Targeting the ubiquitin-proteasome system (UPS) and ubiquitin-like signalling systems (UBL) has been considered a promising therapeutic strategy to treat cancer, neurodegenerative and immunological disorders. There have been multiple efforts recently to identify novel compounds that efficiently modulate the activities of different disease-specific components of the UPS-UBL. However, it is evident that polypharmacology (the ability to affect multiple independent protein targets) is a basic property of small molecules and even highly potent molecules would have a number of "off target" effects. Here we have explored publicly available high-throughput screening data covering a wide spectrum of currently accepted drug targets in order to understand polypharmacology of small molecules targeting different components of the UPS-UBL. We have demonstrated that molecules targeting a given UPS-UBL protein also have high odds to target a given off target spectrum. Moreover, the off target spectrum differs significantly between different components of UPS-UBL. This information can be utilized further in drug discovery efforts, to improve drug efficiency and to reduce the risk of potential side effects of the prospective drugs designed to target specific UPS-UBL components.

\section{INTRODUCTION}

The ubiquitin-proteasome system (UPS) is essential for the turnover and biological function of most proteins [1-3]. In short, ubiquitin is a protein with 76 amino acids, which is activated in an ATP-dependent manner by a ubiquitin-activating enzyme commonly referred to as E1. In the next step ubiquitin is transferred by an ubiquitinconjugating enzyme (commonly referred to as E2) that, together with an ubiquitin-protein ligase (commonly referred to as E3), specifically attaches ubiquitin to a target protein through the amino group of a lysine residue [4]. The targeted protein can be polyubiquitylated (the addition of several ubiquitins to a single lysine residue in a protein) or multiubiquitylated (addition of single or several ubiquitin(s) to several lysines in one protein). Initially, protein ubiquitylation was considered only in the context of signalling for $26 \mathrm{~S}$ proteasome-mediated degradation of the targeted protein (the case of polyubiquitination) [5]. Our current understanding, however, is much wider: there are diverse, nondegradative functions of protein ubiquitylation including the regulation of DNA repair,transcription, mRNA metabolism and splicing [1, 6-10]. Moreover, the $26 \mathrm{~S}$ proteasome is subject to ubiquitination by itself, which affects its activity [11]. In addition, protein ubiquitylation is regulated tightly by complex mechanisms and is a reversible process: ubiquitin molecules can be removed by deubiquitylating enzymes (DUBs) [12].

Recently a number of regulatory cascades very similar to UPS have been discovered, the ubiquitin like modifiers (UBL) $[13,14]$. By marking proteins with ubiquitin-like conjugates, the cell regulates activity of multiple downstream proteins and, thus, controls many important regulatory pathways. The structure of enzymological reactions that are involved in protein 
Table 1: High-throughput screening assays publicly available in the PubChem database modelling different components of ubiquitin or ubiquitin-like cascades.

\begin{tabular}{|c|c|c|c|}
\hline Assay ID & Assay Title & $\begin{array}{c}\text { Active } \\
\text { Molecules }\end{array}$ & $\begin{array}{c}\text { Screened } \\
\text { Molecules }\end{array}$ \\
\hline 485273 & uHTS identification of UBC13 Polyubiquitin Inhibitors via a TR-FRET \\
Assay & 1540 & 328071 \\
\hline 588478 & A screen for small molecule inhibitors of the human deubiquitinating \\
enzyme, UCH37 & 1078 & 329843 \\
\hline 602429 & uHTS identification of SUMO1-mediated protein-protein interactions & 1206 & 362962 \\
\hline 2716 & $\begin{array}{c}\text { Luminescence Microorganism Primary HTS to Identify Inhibitors of the } \\
\text { SUMOylation Pathway Using a Temperature Sensitive Growth Reversal } \\
\text { Mutant Mot1-301 }\end{array}$ & 3324 & 315446 \\
\hline 2006 & uHTS HTRF assay for identification of inhibitors of SUMOylation & 1039 & 289855 \\
\hline 2540 & $\begin{array}{c}\text { HTS Luminescent assay for identification of inhibitors of Sentrin-specific } \\
\text { protease 8 (SENP8) }\end{array}$ & 4122 & 326358 \\
\hline 2599 & $\begin{array}{c}\text { uHTS Luminescent assay for identification of inhibitors of Sentrin-specific } \\
\text { protease 6 (SENP6) }\end{array}$ & 5820 & 324660 \\
\hline 434973 & $\begin{array}{c}\text { uHTS Luminescent assay for identification of inhibitors of Sentrin-specific } \\
\text { protease 7 (SENP7) }\end{array}$ & 4906 & 326853 \\
\hline 602440 & $\begin{array}{c}\text { uHTS Fluorescent Assay Using Nedd8 Protein Substrate for Identification } \\
\text { of Inhibitors of Sentrin-Specific Protease 8 (SENP8) }\end{array}$ & 2342 & 361826 \\
\hline 624204 & $\begin{array}{c}\text { uHTS identification of small molecule inhibitors of the catalytic domain } \\
\text { of the SUMO protease, SENP1 in a FRET assay }\end{array}$ & 774 & 363394 \\
\hline
\end{tabular}

modifications by ubiquitylation is analogous to that used by ubiquitin-like cascades. One of the best studied ubiquitin-like proteins is small ubiquitin-related modifiers SUMO [15]. The conjugation of SUMO to target proteins regulates cell-cycle control, nuclear transport and the response to viral infections [16]. Three SUMO isoforms are currently known in man with functionally distinct roles. At present, about a dozen different ubiquitin-like cascades have been described with varying degrees of completeness [17].

Destabilization of the normal UPS function as well as defects in functionality of UBL signalling cascades have been shown to be linked to multiple pathological conditions such as cancer and neurodegenerative disorders $[17,18]$. Although our understanding of the role of the UPS-UBL in various complex diseases is far from complete, there are examples of successful drug developments targeting the system. Bortezomib is the first drug targeting the UPS (inhibition of the proteasome) that was approved by the FDA for treatment of multiple myeloma in 2003 [19]. The selectivity of proteasome inhibition for killing tumour versus normal cells (normal cells also experience some toxic effects due to abnormal function of the proteasome) by Bortezomib was somewhat surprising. The commonly accepted explanation for this is that tumour cells (in particular white blood cells normally responsible for producing antibodies) have higher concentrations of aberrant proteins that are constantly degraded by the UPS making them more sensitive to the effects of proteasome inhibition $[17,18]$.

The success of Bortezomib along with our increasing understanding of the UPS-UBL and its potential role in human diseases has provoked significant interest in the development of drugs that can target specific components of the UPS-UBL pathways, thus modifying UPS-UBL function [18]. The current trend is the development of compounds targeting specific proteins of the UPS [20]. There have been multiple efforts recently to develop highthroughput screening (HTS) assays to identify drugs that modulate the activity of different components of ubiquitin or ubiquitin-like cascades [21-23]. In the public domain, we were able to find more than a dozen of such HTS (see Table 1), with each reporting thousands of molecules to be efficient modulators and, therefore, each reporting thousands of potential drug leads to act on specific UPS or UBL components.

The current process of drug discovery and subsequent (pre-)clinical development involves several stages; identification of molecules that act on a specific target is only the first step in this timely process. In the next step selected drug candidates are subjected to various tests to investigate potential off targets which would predefine their specific toxicity or side effects [24]. On the one hand, selection of many drug candidates obviously increases the chances to find the one with optimized safety parameters; on the other hand, testing many drug candidates towards a whole spectrum of potential off targets is virtually impossible. At this stage, it is imperative to introduce some rationale for selecting potential risk factors (such as defining the most probable off target effects) and, therefore, to optimize the number of tests needed. This rationale could be introduced by 
Table 2: Top off targets for molecules inhibiting UBC13 (based on data from assay "uHTS identification of UBC13 Polyubiquitin Inhibitors via a TR-FRET Assay").

\begin{tabular}{|c|c|c|c|c|c|c|}
\hline (Target): activity & Odds & $\boldsymbol{k} \boldsymbol{A}^{*}$ & $\begin{array}{l}\text { (The } \\
\text { Number } \\
\text { of Active } \\
\text { molecules) }\end{array}$ & $\boldsymbol{k} \boldsymbol{B} * *$ & $\begin{array}{c}\boldsymbol{K} \boldsymbol{B} * * * \\
\text { (The Number } \\
\text { of InActive } \\
\text { molecules) }\end{array}$ & $\boldsymbol{P}$-value \\
\hline (MAP4K2):inhibitor:mutant & 53.06 & 231 & 1538 & 743 & 223822 & $<1.57 \mathrm{E}-280$ \\
\hline (APAF1):inhibitor & 41.71 & 406 & 1538 & 1908 & 223822 & $<1.57 \mathrm{E}-280$ \\
\hline (RAD52):inhibitor & 36.35 & 140 & 1538 & 615 & 223822 & $1.24 \mathrm{E}-152$ \\
\hline (MAP4K2):inhibitor & 27.8 & 395 & 1538 & 2748 & 223822 & $<1.57 \mathrm{E}-280$ \\
\hline (RAD54L):inhibitor & 20.16 & 55 & 1538 & 411 & 223822 & $3.08 \mathrm{E}-49$ \\
\hline (ATXN2):inhibitor & 19.95 & 221 & 1538 & 1867 & 223822 & $1.24 \mathrm{E}-186$ \\
\hline (MLLT3)[AF4 peptide]:inhibitor & 19.54 & 118 & 1538 & 948 & 223822 & $3.21 \mathrm{E}-101$ \\
\hline [Peg3 Promoter]:inhibitor & 18.08 & 389 & 1538 & 4114 & 223822 & $2.93 \mathrm{E}-302$ \\
\hline (MITF):inhibitor & 16.78 & 202 & 1538 & 1999 & 223822 & $7.99 \mathrm{E}-158$ \\
\hline (PREPL):inhibitor & 12.14 & 153 & 1538 & 2018 & 223822 & $4.28 \mathrm{E}-102$ \\
\hline (PPP1CA):inhibitor & 10.24 & 164 & 1538 & 2578 & 223822 & $1.49 \mathrm{E}-98$ \\
\hline
\end{tabular}

* $\quad k A-$ the number of Active molecules known to have off target activity

** $k B$ - the number of InActive molecules known to have off target activity

$* * *$ Molecules that have no experimentally validated targets are not accounted in the table

mining of publicly available HTS screens covering a wide spectrum of potential drug targets in various complex diseases.

In this article we are going to review several recently published high-throughput screening assays that aimed to identify potential drug leads targeting different components of the UPS-UBL (see Table 1). Furthermore, we provide comparative analyses of these screens versus the large number $(>1000)$ of HTS targetoriented screens publicly available in the PubChem repository. The possibility of such comparison arises from the lucky coincidence that the chemical libraries used in most of these screens are very similar, i.e. screens done independently by different labs with biologically independent assays (targets) share a significant number of the same screened molecules. This allows one to compute for UPS (UBL) targets the cross target correlation versus the spectrum of other targets available in PubChem: the odds of molecules to target any protein from the spectrum if the molecule targets the UPS (UBL). Therefore, for each UPS (UBL) target we obtain a list of top off targets which have high odds to be targeted by potential drug leads. We also provided analyses of discovered top potential off targets on the subject of drug efficiency and drug safety issues.

\section{Inference of Off target spectrum for UPS (UBL) targets}

PubChem BioAssay [25] repository stores the results of biochemical HTS target oriented assays. Based on the
PubChem BioAssay data model, results of HTS target oriented assays, in simple terms, could be interpreted as a subset of small molecules reported to inhibit (in rare cases activate) the targeted protein. The abundance of this information ( $>1000$ independent assays) from the PubChem BioAssay repository could be integrated on a large scale to derive binding spectra for approximately half of the million molecules across several hundred proteins. Many of these molecules have been tested in UPS (UBL) assays. Let us denote KA to be the number of active molecules (molecules exhibiting activity in the assay at relevant concentrations) in the HTS UPS (UBL) assay and as KB the number of inactive molecules. For each off target protein " $Z$ " we count the number $(k A)$ of the active molecules (from the UPS assay) which target " $Z$ " (have been reported in the other HTS assay as active versus " $Z$ ") and $k B$ is the number of inactive molecules which target $\mathrm{Z}$. Odds for the molecules (to be active) targeting " $Z$ " is ( $k A$ / $k B$ ) while the same odds for the molecules which do not target " $\mathrm{Z}$ " is $((K A-k A) /(\mathrm{KB}-\mathrm{kB}))$. The odds ratio is $(k A / k B)$ / $((K A-k A) /(K B-k B))$ and indicates the increase/decrease of odds for a molecule to target " $Z$ " while targeting the UPSUBL protein. Significance of the odds ratio is computed using $X^{2}$ - distribution and the adjustment of $\mathrm{p}$-values for multiple testing (each target one hypothesis) is done using the FDR procedure [26-29].

\section{UBC13 Polyubiquitin Inhibitors}

Ubc13 is an unusual E2 ubiquitin-conjugating enzyme which is only active as a heterodimer with another 
E2 enzyme and produces polyubiquitin chains exclusively linked at lysine residue 63 of ubiquitin. Ubc13 specifically ubiquitinates Tumor Necrosis Factor Receptor-Associated Factors (TRAFs), a family of adapter proteins. Ubc13mediated ubiquitination of TRAFs is recognized as a critical step in signalling by TNFRs during the innate and acquired immune responses [30]. Inhibition of Ubc13 is considered as a potential strategy for development of novel immunosuppressive, anti-inflammatory agents as well as agents for treatment of neurodegenerative diseases [31]. In vitro high-throughput screening assays based on the principal of time-resolved fluorescence resonance energy transfer (TR-FRET) have been developed to identify potential inhibitors of Ubc13 activity [32]. Terbium-ubiquitin and fluorescein-ubiquitin have been used to generate a FRET reaction. In total, a library of 328 071 compounds were screened and 1540 were reported as active (efficiently inhibiting Ubc13 at clinically relevant concentrations).

The top ten potential off target activities for the molecules inhibiting Ubc13 are presented in Table 2. For example, the ability of a molecule to inhibit Ubc13 significantly increases the odds ( 40 fold) for the molecule to additionally inhibit APAF1. In total, 2314 (406 + 1908) molecules which were tested in the Ubc13 screen demonstrated the potential to inhibit APAF1 and 406 of them exhibited potent inhibition of Ubc13 while 1908 did not. Observed odds for a molecule to inhibit Ubc13 in the screen is $\sim 0.006(1538 / 223822)$, while observed odds for the molecules experimentally validated to inhibit APAF1 is approximately 40 times higher $\sim 0.21(406 / 1908)$. Thus, we observe a strong association between Ubc13 and APAF1: molecules targeting one protein have a reasonable chance to target the other as an off target.

Recent evidence has implicated the E3 ligase activity of TRAFs in the pathogenic aggregation of mutant proteins in neurodegenerative diseases such as Huntington disease [33]. Instead of conventional polyubiquitination, TRAF6 promotes atypical ubiquitination (with the Ubc13 as the E2) of mutated misfolded proteins and, thereby, prevents them from degradation $[34,35]$. Recently APAF1 dominant negative inhibition was tested for its antiapoptotic effect on degenerating nigrostriatal neurons in a 1-methyl-4-phenyl-1,2,3,6-tetrahydropyridine (MPTP) model of Parkinson's disease and was shown to inhibit MPTP toxicity [36]. Thus, both Ubc13 and APAF1 are potential therapeutic targets in Parkinson's disease but with different mechanism of action. Our analysis reveals that Ubc13 and APAF1 are frequently targeted together by small molecules as observed from the available HTS screens. This makes the Ubc13 and APAF1 pair an attractive multitarget for development of therapeutics with polypharmacological mechanisms of action in Parkinson's disease.

\section{Inhibitors of the human deubiquitinating enzyme (UCH37)}

Deubiquitinating enzymes (DUB) represent a group of cysteine proteases that cleave the isopeptide bond between ubiquitin and its conjugated proteins [37]. The HTS assay (PubChem Id 588478) aims to identify small molecule inhibitors of human UCH37, which is tightly associated with the proteasome. Specifically, this screen sought to identify small molecules that inhibit the increase in fluorescence resulting from the UCH37 mediated cleavage of a fluorescent substrate, ubiquitin-7-amido-4methylcoumarin (Ub-AMC). In total, a library of 329843 small molecules was screened and 1078 molecules were reported as active (efficiently inhibiting $\mathrm{UCH} 37$ at low concentration).

The top ten potential off target activities for the molecules inhibiting UCH37 are presented in Table 3. For example, the ability of a molecule to inhibit UCH37 significantly increases the odds ( 30 fold) for the molecule to inhibit TDP2 (tyrosyl-DNA phosphodiesterase 2). In total, $965(119+846)$ molecules tested in the assay have been reported in other HTS screens as efficient inhibitors of TDP2 and 119 of them demonstrated potent inhibition of UCH37 in the assay. Observed odds for a molecule to inhibit UCH37 in the screen is $\sim 0.005$ (2130/ 238331), while observed odds for the molecules known to inhibit TDP2 is approximately 30 times higher $\sim 0.14$ $(119 / 846)$. Thus, we observe strong association between UCH37 and TDP2: molecules targeting one protein have a reasonable chance to target the other as an off target.

Human USPs are considered as novel targets for therapeutic intervention in a number of human cancers, including prostate, colon and breast cancer as well as acute lymphoblastic leukemia [38]. TyrosylDNA phosphodiesterase 2 (TDP2) is implicated in topoisomerase-mediated repair of DNA damage. As a drug target, TDP2 is hypothesized to mediate drug resistance to topoisomerase II inhibition by etoposide [39]. Therefore, inhibition of TDP2 is proposed as a promising approach to overcome intrinsic or acquired resistance to topo IItargeted drug therapy. The observed properties of screened small molecules to inhibit both targets could be exploited for the design of multitarget agents. Considering that the mechanisms of drug action for UCH37 and TDP2 differ significantly, the potential for drugs targeting both of them is likely to be more efficient than targeting independent mechanisms in the cancer cell. Therefore, multi-target drugs affecting several cancer mechanisms represented by UCH37 and TDP2 could be more efficient. On the other hand, TDP2 has been reported to be essential for normal neural function and is required to maintain normal levels of several gene transcripts in the mouse brain during development [40]. Therefore, targeting TDP2 has increased odds to be associated with neurological side effects. 
Table 3: Top off targets for molecules inhibiting UCH37 (based on data from assay "A screen for small molecule inhibitors of the human deubiquitinating enzyme, UCH37").

\begin{tabular}{|c|c|c|c|c|c|c|}
\hline (Target): activity & $\begin{array}{c}\text { Odds } \\
\text { Ratio }\end{array}$ & $k A^{*}$ & \begin{tabular}{|l|}
\multicolumn{1}{c|}{$K A$} \\
(The \\
Number \\
of Active \\
molecules)
\end{tabular} & $k B^{* *}$ & $\begin{array}{c}K B * * * \\
\text { (The Number } \\
\text { of InActive } \\
\text { molecules) }\end{array}$ & $P$-value \\
\hline (USP1):inhibitor & 69.85 & 158 & 1074 & 508 & 206210 & 4.73E-211 \\
\hline (PAFAH1B3):inhibitor & 55.05 & 140 & 1074 & 560 & 206210 & $3.35 \mathrm{E}-175$ \\
\hline (TDP2):inhibitor & 30.25 & 119 & 1074 & 846 & 206210 & $3.14 \mathrm{E}-122$ \\
\hline (HKDC1):inhibitor & 30.11 & 55 & 1074 & 369 & 206210 & $5.08 \mathrm{E}-58$ \\
\hline (APAF1):inhibitor & 24.98 & 181 & 1074 & 1660 & 206210 & $4.65 \mathrm{E}-169$ \\
\hline (WHSC1):inhibitor & 21.86 & 124 & 1074 & 1224 & 206210 & $2.06 \mathrm{E}-111$ \\
\hline (CASP6):inhibitor & 20.33 & 115 & 1074 & 1209 & 206210 & $2.07 \mathrm{E}-100$ \\
\hline [RBBP9]:inhibitor & 19.4 & 61 & 1074 & 638 & 206210 & $1.27 \mathrm{E}-53$ \\
\hline (CTSL1):inhibitor & 18.09 & 98 & 1074 & 1138 & 206210 & $1.07 \mathrm{E}-81$ \\
\hline [FadD28]:competitors for binfing & 17.23 & 52 & 1074 & 607 & 206210 & $1.20 \mathrm{E}-43$ \\
\hline
\end{tabular}

* $\quad k A$ - the number of Active molecules known to have off target activity

** $\quad k B$ - the number of InActive molecules known to have off target activity

*** Molecules that have no experimentally validated targets are not accounted in the table

\section{SUMO-lation assays}

Targeting SUMOylation has become an important drug discovery trend considering the number of HTS assays developed (see table 1). Targeting SUMOylation has been considered as a drug discovery strategy to overcome cancer cell resistance to chemo and radiation therapy [41]. In particular, some studies specifically search for small molecule inhibitors of protein-protein interactions mediated by SUMO. The rationale in developing an HTS assay "identification of SUMO1mediated protein-protein interactions" was a demand for discovery of small molecules that can specifically act as "chemical modulators" of SUMO-mediated signaling. The assay was developed on the principal of timeresolved fluorescence resonance energy transfer (TRFRET) based on a N-terminal-fluorescein tagged-SUMO 1 specific sequence peptide. The binding event brings the fluorescein acceptor moiety in close proximity to the Tbdonor to allow time-resolved lanthanide fluorescence from the terbium. In total, a library of 362962 small molecules was screened and 1206 molecules were reported as active (efficiently inhibiting SUMO1-mediated protein-protein interactions (PPI) at low concentrations).

The top ten potential off target activities for the small molecule inhibitors of PPI mediated by SUMO are presented in Table 4 . For example, the ability of a molecule to inhibit MBD2 significantly increases odds ( 110 fold) for the molecule to inhibit SUMO1-mediated PPI. In total, $258(82+176)$ molecules tested in the assay have been reported in other HTS screens as efficient inhibitors of MBD2 and 82 of them demonstrated potent inhibition of SUMO1-mediated PPI. Observed odds for a molecule to inhibit SUMO1-mediated PPI in the screen is $\sim 0.0045(1091 / 241605)$, while observed odds for the molecules known to inhibit MBD2 is approximately 100 times higher $\sim 0.45(82 / 176)$. Thus, we observe a strong association between MBD2 and SUMO1-mediated PPI: again, molecules targeting one have a reasonable chance to target the other one as an off target.

Among the top potential off targets for the molecules inhibiting SUMO1-mediated PPI we can see proteins commonly associated as drug targets against cancer. For example, MBD2 belongs to the family of methyl$\mathrm{CpG}$ binding proteins [42]. MBD2 mediates epigenetic gene silencing and thus is an attractive target in cancer treatment for reactivation of apoptotic genes as a primary therapeutic mechanism. Another off target WRN is RecQ DNA helicase that participates in suppression of DNA hyper-recombination and repair. WRN was considered as a cancer therapeutic target in hypopharyngeal carcinomas, which have the worst prognosis among head and neck squamous cell carcinomas (HNSCC) with a rapidly rising incidence [43]. It has been shown that WRN is highly expressed in HNSCC, and that siRNA-mediated silencing of the gene suppressed carcinoma cell growth in vitro. Therefore, there are several potential off targets for the molecules inhibiting SUMO1-mediated PPI which are potential anticancer targets. As has been already mentioned, this knowledge could be utilized for the development of multitarget drugs and which may therefore provide therapeutic intervention affecting multiple independent anticancer mechanisms.

On the other hand, there are two potential off targets which seem better avoided. The HKDC1 (HexoKinase 
Table 4: Top off targets for molecules inhibiting SUMO1-mediated PPI (based on data from assay "uHTS identification of SUMO1-mediated protein-protein interactions").

\begin{tabular}{|c|c|c|c|c|c|c|}
\hline (Target): activity & $\begin{array}{c}\text { O d d s } \\
\text { Ratio }\end{array}$ & $\boldsymbol{k} \boldsymbol{A}^{*}$ & $\begin{array}{c}\text { (The Num- } \\
\text { ber of Ac- } \\
\text { tive mol- } \\
\text { ecules) }\end{array}$ & $\boldsymbol{k B} * * \begin{array}{c}\boldsymbol{K} \boldsymbol{B} * * * \\
\text { (The Number } \\
\text { InActive } \\
\text { molecules) }\end{array}$ & $\boldsymbol{P}$-value \\
\hline (MMP2):inhibitor & 144.62 & 68 & 1091 & 111 & 241605 & $4.66 \mathrm{E}-111$ \\
\hline (MBD2):inhibitor & 111.48 & 82 & 1091 & 176 & 241605 & $4.35 \mathrm{E}-126$ \\
\hline (ACP1):inhibitor & 105.01 & 224 & 1091 & 593 & 241605 & $<1.57 \mathrm{E}-280$ \\
\hline (PTPN5):inhibitor & 88.51 & 216 & 1091 & 672 & 241605 & $<1.57 \mathrm{E}-280$ \\
\hline (BLM):inhibitor & 85.02 & 87 & 1091 & 246 & 241605 & $3.85 \mathrm{E}-125$ \\
\hline (HKDC1):inhibitor & 73.78 & 125 & 1091 & 423 & 241605 & $1.17 \mathrm{E}-171$ \\
\hline (WRN):inhibitor & 69.34 & 314 & 1091 & 1400 & 241605 & $<1.57 \mathrm{E}-280$ \\
\hline (DNMT1):inhibitor & 69.02 & 459 & 1091 & 2516 & 241605 & $<1.57 \mathrm{E}-280$ \\
\hline (RAPGEF3):antagonist & 66.59 & 108 & 1091 & 398 & 241605 & $9.99 \mathrm{E}-145$ \\
\hline
\end{tabular}

* $\quad k A-$ the number of Active molecules known to have off target activity

** $k B$ - the number of InActive molecules known to have off target activity

*** Molecules that have no experimentally validated targets are not accounted in the table

Domain Containing I) gene encodes mammalian hexokinase. Hexokinases (HKs) catalyse the first step in glucose metabolism and play a major role in regulating the metabolic fate of glucose in the tissues where they are expressed. The dysregulation of glucose is commonly associated with Type 2 diabetes, and activation of HKDC1 is considered as a potential target for diabetes therapeutics while inhibition of HKDC1 could lead to the undesired inhibition of glucose metabolism [44]. Rap guanine nucleotide exchange factor (the gene is known as RAPGEF3, but the protein is usually referred to as EPAC) is closely involved in the regulation of cAMP signaling. EPAC has been implicated in playing important roles in major human pathological conditions such as diabetes and heart disease and EPAC is now considered as a new regulator of cardiac physiopathology [45]. Although its effects are much less well known than the classical cAMP effector, PKA, several studies have investigated the cardiac role of EPAC, providing evidence that EPAC modulates intracellular $\mathrm{Ca}(2+)$ levels. In one of the first analyses, it was shown that EPAC can increase the frequency of spontaneous $\mathrm{Ca}(2+)$ oscillations in cultured rat cardiomyocytes. Considering that drugs targeting EPAC have increased chances to lead to unexpected cardiac side effects, it would be better to select drug leads with SUMO1-mediated PPI activities that do not possess activity towards RAPGEF3 gene products to minimize these risks.

\section{SENP assays}

A group of proteases known as SENPs are involved in both the maturation of SUMO precursors (endopeptidase cleavage) and deconjugation of the targets (isopeptidase cleavage) [46]. Human cells contain seven SENPs (SENPs -1, -2, -3, -5, -6, -7, and -8), and several of these have been characterized as SUMO (or Nedd8) endopeptidases or isopeptidases [47]. The importance of SENPs as drug targets is emphasised by the evidence that SUMOlation regulates cell fate decisions by regulating p53, mdm2, and PML activity [41, 48]. A number of assays covering SENP1, SENP6, SENP7 and SENP8 have been developed recently (see table 1). The objective of the assays was to identify small molecule inhibitors specific for SENP6, SENP7 and SENP8. The assays utilized an RLRGG-aminoluciferin peptide substrate and used SENP8-dependent deconjugation of the aminoluciferin, which serves as a substrate for the coupled luciferase. In total, libraries covering $\sim 300000$ small molecules were screened versus the SENP family and several thousand molecules were reported as active (efficiently inhibiting SENP proteases at low concentration). Table 5 reports the top 10 ten potential off target activities for the small molecule inhibitors of SENP6.

The ability of a molecule to inhibit SENP6 observed in the assay was very strongly correlated with the ability of the molecule to inhibit other SENP family members: SENP7 and SENP8. This could be partially explained by technical bias. The assays are based on Caspase-3 dependent deconjugation of the aminoluciferin, which serves as a substrate for the coupled Ultra-GloTM luciferase. Therefore, molecules inhibiting CASP3 will also provide an artefactual signal in the assay. For this reason several control assays have been carried out in order to deprioritize compounds that inhibit Caspase-3. As it can be seen from table 5 , the majority of molecules detected in the initial screen for potential SENP inhibitors are also Caspase- 3 inhibitors. Considering the numbers, 
Table 5: Top off targets for molecules inhibiting SENP6 (based on data from assay "uHTS Luminescent assay for identification of inhibitors of Sentrin-specific protease 6 (SENP6)").

\begin{tabular}{|l|l|l|l|l|l|l|}
\hline (Target): activity & $\begin{array}{l}\text { Odds } \\
\text { Ratio }\end{array}$ & $\boldsymbol{k} \boldsymbol{A}^{*}$ & $\begin{array}{l}\boldsymbol{K} \boldsymbol{A} \\
\text { (The } \\
\text { Number Active } \\
\text { of } \\
\text { molecules) }\end{array}$ & $\boldsymbol{k} \boldsymbol{B} * *$ & $\begin{array}{l}\boldsymbol{K} \boldsymbol{B} * * \\
\text { (The Number } \\
\text { of InActive } \\
\text { molecules) }\end{array}$ & $\boldsymbol{P}$-value \\
\hline (SENP7):inhibitor & 1156.49 & 4904 & 5779 & 1059 & 219581 & $<1.57 \mathrm{E}-280$ \\
\hline (CASP3):inhibitor & 1111.03 & 3950 & 5779 & 426 & 219581 & $<1.57 \mathrm{E}-280$ \\
\hline (SENP8):inhibitor & 459.86 & 4734 & 5779 & 2142 & 219581 & $<1.57 \mathrm{E}-280$ \\
\hline (PKM):inhibitor & 138.06 & 107 & 5779 & 30 & 219581 & $1.57 \mathrm{E}-141$ \\
\hline (MMP14):inhibitor transcription & 30.53 & 225 & 5779 & 291 & 219581 & $1.07 \mathrm{E}-211$ \\
\hline (TNFRSF10B):inhibitor & 27.9 & 1148 & 5779 & 1934 & 219581 & $<1.57 \mathrm{E}-280$ \\
\hline (NPC1):activator & 27.67 & 2286 & 5779 & 5074 & 219581 & $<1.57 \mathrm{E}-280$ \\
\hline (RAB9A):activator & 26.6 & 2550 & 5779 & 6331 & 219581 & $<1.57 \mathrm{E}-280$ \\
\hline (STAT3):inhibitor & 23.94 & 572 & 5779 & 1003 & 219581 & $<1.57 \mathrm{E}-280$ \\
\hline $\begin{array}{l}\text { (TP53):re-activators of p53 using a } \\
\text { Luc reporter }\end{array}$ & 23.47 & 105 & 5779 & 173 & 219581 & $2.25 \mathrm{E}-91$ \\
\hline
\end{tabular}

* $\quad k A-$ the number of Active molecules known to have off target activity

** $\quad k B-$ the number of InActive molecules known to have off target activity

*** Molecules that have no experimentally validated targets are not accounted in the table

we can see that more than 80 percent of active molecules are non-specific in relation to either CASP3 or other family members.

As in previous cases, there a number of off target activities which can be utilized for development of multitarget drugs as well as some specific off targets which are better to be avoided. For example, the molecules inhibiting the SENP family seem to have increased odds ( $\sim 23$ fold) to reactivate $\mathrm{p} 53$. Lack of $\mathrm{p} 53$ expression or expression of mutant p53 is common in human cancers and is associated with increased tumor growth and resistance to therapies $[49,50]$. Significant efforts toward pharmaceutical reactivation of defective p53 by small molecules are considered as an independent anticancer therapeutic strategy [51-54]. Indeed, reactivated p53 can lead to tumor destruction [55]. Therefore, selecting drug candidates for the next developmental stages from SENPs screens where reactivation of p53 is a potential off target activity would increase the chances of developing potent anticancer agents that target independent anticancer mechanisms.

On the other hand, those molecules inhibiting the SENP family seem to have increased odds ( 140 fold) to inhibit PKM (pyruvate kinase, muscle). Pyruvate kinase is a key glycolytic enzyme which is expressed as several isoforms in many cell types (liver, pancreatic cells, small intestine and so on). PKM has been reported to have important roles in many diseases including cancer and PKM polymorphisms have been shown to be associated with type 2 diabetes [56]. Considering the complicated and controversial role of PKM in human diseases, it would be better to avoid potential drug leads that additionally target PKM.

\section{DISCUSSION}

There have been significant efforts in recent years to discover small molecular potent inhibitors of ubiquitin-proteasome or ubiquitin-like signalling systems including a number of high-throughput screening projects which reported thousands of potential drug lead molecules efficiently targeting disease specific UPS-UBL components in vitro [18, 20-22]. A critical step in drug discovery is a selection of those drug leads that have better chances of success in clinical trials [57]. Selected molecules are subjected to a number of preclinical tests to address potential issues with pharmacodynamics, pharmacokinetics, ADME properties and potential toxicity. Considering the low rate of success in current drug discovery, any rationale for improving the selection process of drug leads that increases the chances of avoiding further safety [58] or efficacy issues [59-61] is of paramount importance.

It is now commonly accepted that polypharmacology (the ability to affect multiple independent protein targets) is a basic property of small molecules $[60,62-$ 64]. Therefore, understanding the potential off target spectrum of molecules targeting UPS(UBL) is of practical importance $[65,66]$. Here we have explored the abundance of publicly available high-throughput screening assay data in order to understand polypharmacology of small molecules tested in UPS-UBL high-throughput 
screens (Table 1). To do this, we interrogated $(>1000)$ HTS target oriented screens available in the public domain (PubChem repository [25]), which cover a wide spectrum of commonly accepted drug targets for various diseases. The opportunity of such statistical analysis stems from the fact that the chemical libraries used in most of these highthroughput screens share a significant number of common molecules. Thus we computed for each UPS(UBL) target the off target spectrum by counting the number of molecules which are reported to be active/inactive between a pair of assays (UPS-UBL assay versus off target assay) and thus computed the shift in odds for a molecule to target a specific component of the UPS while being experimentally validated to target another given protein. For each UPS(UBL) target we derived the list of top off targets (off target spectrum) which have high odds to be targeted by potential UPS(UBL) drug leads.

Polypharmacology of a small molecule could be either beneficial (co-targeting of certain proteins could increase efficiency) or the opposite (co-targeting of certain proteins could lead to undesired side effects). Here we have demonstrated that information for potential off targets for molecules targeting different UPS components could be utilized to design multi-target drugs, which are able to affect independent disease specific mechanisms. For example, from the HTS data we observed a strong correlation between drugs targeting Ubc13 and APAF1. Both targets could be potentially implicated in treatment of the pathogenic aggregation of mutant proteins in neurodegenerative diseases such as Huntingtons disease, although targeting different mechanisms. This observation (pharmacological association between Ubc13 and APAF1) can be utilized in future efforts to identify potent drug leads that inhibit both targets and, therefore, by affecting two independent mechanisms in neurodegenerative diseases the potential efficacy of the multitarget therapy is enhanced.

Finally, we have demonstrated that information for potential off targets for molecules targeting different UPS components could be utilized to reduce risks associated with potential side effects of drug leads. Among pharmacologically associated off targets for different UPS targets there are multiple proteins known to be associated with various disease conditions such as cardiovascular or diabetes. For design of anticancer agents, for example, these types of off targets should be avoided as inhibition or activation of these proteins might have undesirable corresponding side effects.

\section{ACKNOWLEDGMENTS}

This work was supported by the UK Medical Research Council (MRC) and in part by the Program of Russian Science Foundation (to AL).

\section{CONFLICTS OF INTEREST}

There is no conflict of interest.

\section{REFERENCES}

1. Welchman RL, Gordon C and Mayer RJ. Ubiquitin and ubiquitin-like proteins as multifunctional signals. Nature reviews Molecular cell biology. 2005; 6:599-609.

2. Blagosklonny $\mathrm{MV}, \mathrm{Wu}$ GS, Omura $\mathrm{S}$ and ElDeiry WS. Proteasome-dependent regulation of p21(WAF1/ CIP1) expression. Biochemical and biophysical research communications. 1996; 227:564-569.

3. Blagosklonny MV. Do VHL and HIF-1 mirror p53 and Mdm-2? Degradation-transactivation loops of oncoproteins and tumor suppressors. Oncogene. 2001; 20:395-398.

4. Hershko A, Heller H, Elias $\mathrm{S}$ and Ciechanover A. Components of ubiquitin-protein ligase system. Resolution, affinity purification, and role in protein breakdown. The Journal of biological chemistry. 1983; 258:8206-8214.

5. Thrower JS, Hoffman L, Rechsteiner M and Pickart CM. Recognition of the polyubiquitin proteolytic signal. The EMBO journal. 2000; 19:94-102.

6. Bernassola F, Ciechanover A and Melino G. The ubiquitin proteasome system and its involvement in cell death pathways. Cell death and differentiation. 2010; 17:1-3.

7. Melino G. Discovery of the ubiquitin proteasome system and its involvement in apoptosis. Cell death and differentiation. 2005; 12:1155-1157.

8. Mittenberg AG, Moiseeva TN and Barlev NA. Role of proteasomes in transcription and their regulation by covalent modifications. Frontiers in bioscience : a journal and virtual library. 2008; 13:7184-7192.

9. Kulichkova VA, Tsimokha AS, Fedorova OA, Moiseeva TN, Bottril A, Lezina L, Gauze LN, Konstantinova IM, Mittenberg AG and Barlev NA. 26S proteasome exhibits endoribonuclease activity controlled by extra-cellular stimuli. Cell cycle. 2010; 9:840-849.

10. Fedorova OA, Moiseeva TN, Nikiforov AA, Tsimokha AS, Livinskaya VA, Hodson M, Bottrill A, Evteeva IN, Ermolayeva JB, Kuznetzova IM, Turoverov KK, Eperon I and Barlev NA. Proteomic analysis of the 20S proteasome (PSMA3)-interacting proteins reveals a functional link between the proteasome and mRNA metabolism. Biochemical and biophysical research communications. 2011; 416:258-265.

11. Moiseeva TN, Bottrill A, Melino G and Barlev NA. DNA damage-induced ubiquitylation of proteasome controls its proteolytic activity. Oncotarget. 2013; 4:1338-1348.

12. Nijman SM, Luna-Vargas MP, Velds A, Brummelkamp TR, Dirac AM, Sixma TK and Bernards R. A genomic and functional inventory of deubiquitinating enzymes. Cell. 2005; 123:773-786.

13. Johnson ES, Schwienhorst I, Dohmen RJ and Blobel G. The 
ubiquitin-like protein Smt3p is activated for conjugation to other proteins by an Aos $1 \mathrm{p} / \mathrm{Uba} 2 \mathrm{p}$ heterodimer. The EMBO journal. 1997; 16:5509-5519.

14. Gostissa M, Hengstermann A, Fogal V, Sandy P, Schwarz SE, Scheffner M and Del Sal G. Activation of p53 by conjugation to the ubiquitin-like protein SUMO-1. The EMBO journal. 1999; 18:6462-6471.

15. Hunter T and Sun H. Crosstalk between the SUMO and ubiquitin pathways. Ernst Schering Foundation symposium proceedings. 2008; (1):1-16.

16. Tempe D, Piechaczyk M and Bossis G. SUMO under stress. Biochemical Society transactions. 2008; 36:874-878.

17. Nalepa G, Rolfe M and Harper JW. Drug discovery in the ubiquitin-proteasome system. Nature reviews Drug discovery. 2006; 5:596-613.

18. Edelmann MJ, Nicholson $B$ and Kessler BM. Pharmacological targets in the ubiquitin system offer new ways of treating cancer, neurodegenerative disorders and infectious diseases. Expert reviews in molecular medicine. 2011; 13:e35.

19. Richardson PG, Sonneveld P, Schuster MW, Irwin D, Stadtmauer EA, Facon T, Harousseau JL, Ben-Yehuda D, Lonial S, Goldschmidt H, Reece D, San-Miguel JF, Blade J, Boccadoro M, Cavenagh J, Dalton WS, et al. Bortezomib or high-dose dexamethasone for relapsed multiple myeloma. The New England journal of medicine. 2005; 352:24872498.

20. Bedford L, Lowe J, Dick LR, Mayer RJ and Brownell JE. Ubiquitin-like protein conjugation and the ubiquitinproteasome system as drug targets. Nature reviews Drug discovery. 2011; 10:29-46.

21. Rossi M, Rotblat B, Ansell K, Amelio I, Caraglia M, Misso G, Bernassola F, Cavasotto CN, Knight RA, Ciechanover A and Melino G. High throughput screening for inhibitors of the HECT ubiquitin E3 ligase ITCH identifies antidepressant drugs as regulators of autophagy. Cell death \& disease. 2014; 5:e1203.

22. Ungermannova D, Lee J, Zhang G, Dallmann HG, McHenry CS and Liu X. High-throughput screening AlphaScreen assay for identification of small-molecule inhibitors of ubiquitin E3 ligase SCFSkp2-Cks1. Journal of biomolecular screening. 2013; 18:910-920.

23. Huang J, Sheung J, Dong G, Coquilla C, Daniel-Issakani S and Payan DG. High-throughput screening for inhibitors of the e3 ubiquitin ligase APC. Methods in enzymology. 2005; 399:740-754.

24. DiMasi JA, Feldman L, Seckler A and Wilson A. Trends in risks associated with new drug development: success rates for investigational drugs. Clinical pharmacology and therapeutics. 2010; 87:272-277.

25. Wang Y, Bolton E, Dracheva S, Karapetyan K, Shoemaker BA, Suzek TO, Wang J, Xiao J, Zhang J and Bryant SH. An overview of the PubChem BioAssay resource. Nucleic Acids Res. 2010; 38(Database issue):D255-266.
26. Hochberg Y and Benjamini Y. More powerful procedures for multiple significance testing. Statistics in medicine. 1990; 9:811-818.

27. Antonov AV. BioProfiling.de: analytical web portal for high-throughput cell biology. Nucleic acids research. 2011; 39(Web Server issue):W323-327.

28. Dietmann S, Lee W, Wong P, Rodchenkov I and Antonov AV. CCancer: a bird's eye view on gene lists reported in cancer- related studies. Nucleic acids research. 2010; 38:W118-W123.

29. Antonov AV and Mewes HW. Complex functionality of gene groups identified from high-throughput data. Journal of molecular biology. 2006; 363:289-296.

30. Andersen PL, Zhou H, Pastushok L, Moraes T, McKenna S, Ziola B, Ellison MJ, Dixit VM and Xiao W. Distinct regulation of Ubc13 functions by the two ubiquitinconjugating enzyme variants Mms2 and Uev1A. The Journal of cell biology. 2005; 170:745-755.

31. Chang JH, Xiao Y, Hu H, Jin J, Yu J, Zhou X, Wu X, Johnson HM, Akira S, Pasparakis M, Cheng X and Sun SC. Ubc13 maintains the suppressive function of regulatory $\mathrm{T}$ cells and prevents their conversion into effector-like T cells. Nature immunology. 2012; 13:481-490.

32. Madiraju C, Welsh K, Cuddy MP, Godoi PH, Pass I, Ngo T, Vasile S, Sergienko EA, Diaz P, Matsuzawa S and Reed JC. TR-FRET-based high-throughput screening assay for identification of UBC13 inhibitors. Journal of biomolecular screening. 2012; 17:163-176.

33. Xie P. TRAF molecules in cell signaling and in human diseases. Journal of molecular signaling. 2013; 8:7.

34. Zucchelli S, Marcuzzi F, Codrich M, Agostoni E, Vilotti S, Biagioli M, Pinto M, Carnemolla A, Santoro C, Gustincich $\mathrm{S}$ and Persichetti F. Tumor necrosis factor receptorassociated factor 6 (TRAF6) associates with huntingtin protein and promotes its atypical ubiquitination to enhance aggregate formation. The Journal of biological chemistry. 2011; 286:25108-25117.

35. Zucchelli S, Codrich M, Marcuzzi F, Pinto M, Vilotti S, Biagioli M, Ferrer I and Gustincich S. TRAF6 promotes atypical ubiquitination of mutant DJ-1 and alpha-synuclein and is localized to Lewy bodies in sporadic Parkinson's disease brains. Human molecular genetics. 2010; 19:37593770 .

36. Mochizuki H, Hayakawa H, Migita M, Shibata M, Tanaka R, Suzuki A, Shimo-Nakanishi Y, Urabe T, Yamada M, Tamayose K, Shimada T, Miura M and Mizuno Y. An AAV-derived Apaf-1 dominant negative inhibitor prevents MPTP toxicity as antiapoptotic gene therapy for Parkinson's disease. Proceedings of the National Academy of Sciences of the United States of America. 2001; 98:10918-10923.

37. Stone M, Hartmann-Petersen R, Seeger M, BechOtschir D, Wallace M and Gordon C. Uch2/Uch37 is the major deubiquitinating enzyme associated with the $26 \mathrm{~S}$ proteasome in fission yeast. Journal of molecular biology. 
2004; 344:697-706.

38. Fraile JM, Quesada V, Rodriguez D, Freije JM and LopezOtin C. Deubiquitinases in cancer: new functions and therapeutic options. Oncogene. 2012; 31:2373-2388.

39. Raoof A, Depledge P, Hamilton NM, Hamilton NS, Hitchin JR, Hopkins GV, Jordan AM, Maguire LA, McGonagle AE, Mould DP, Rushbrooke M, Small HF, Smith KM, Thomson GJ, Turlais F, Waddell ID, et al. Toxoflavins and deazaflavins as the first reported selective small molecule inhibitors of tyrosyl-DNA phosphodiesterase II. Journal of medicinal chemistry. 2013; 56:6352-6370.

40. Gomez-Herreros F, Schuurs-Hoeijmakers JH, McCormack M, Greally MT, Rulten S, Romero-Granados R, Counihan TJ, Chaila E, Conroy J, Ennis S, Delanty N, CortesLedesma F, de Brouwer AP, Cavalleri GL, El-Khamisy $\mathrm{SF}$, de Vries BB, et al. TDP2 protects transcription from abortive topoisomerase activity and is required for normal neural function. Nature genetics. 2014; 46:516-521.

41. Bartek J and Hodny Z. SUMO boosts the DNA damage response barrier against cancer. Cancer cell. 2010; 17:9-11.

42. Parry L and Clarke AR. The Roles of the Methyl-CpG Binding Proteins in Cancer. Genes \& cancer. 2011; 2:618630.

43. Arai A, Chano T, Futami K, Furuichi Y, Ikebuchi K, Inui T, Tameno H, Ochi Y, Shimada T, Hisa Y and Okabe H. RECQL1 and WRN proteins are potential therapeutic targets in head and neck squamous cell carcinoma. Cancer research. 2011; 71:4598-4607.

44. Hayes MG, Urbanek M, Hivert MF, Armstrong LL, Morrison J, Guo C, Lowe LP, Scheftner DA, Pluzhnikov A, Levine DM, McHugh CP, Ackerman CM, Bouchard L, Brisson D, Layden BT, Mirel D, et al. Identification of HKDC1 and BACE2 as genes influencing glycemic traits during pregnancy through genome-wide association studies. Diabetes. 2013; 62:3282-3291.

45. Ruiz-Hurtado G, Morel E, Dominguez-Rodriguez A, Llach A, Lezoualc'h F, Benitah JP and Gomez AM. Epac in cardiac calcium signaling. Journal of molecular and cellular cardiology. 2013; 58:162-171.

46. Drag M and Salvesen GS. DeSUMOylating enzymes-SENPs. IUBMB life. 2008; 60:734-742.

47. Kolli N, Mikolajczyk J, Drag M, Mukhopadhyay D, Moffatt N, Dasso M, Salvesen G and Wilkinson KD. Distribution and paralogue specificity of mammalian deSUMOylating enzymes. The Biochemical journal. 2010; 430:335-344.

48. Drag M, Mikolajczyk J, Krishnakumar IM, Huang Z and Salvesen GS. Activity profiling of human deSUMOylating enzymes (SENPs) with synthetic substrates suggests an unexpected specificity of two newly characterized members of the family. The Biochemical journal. 2008; 409:461-469.

49. Azmi AS. Pharmaceutical reactivation of $\mathrm{p} 53$ pathways in cancer. Current pharmaceutical design. 2011; 17:534-535.

50. Amelio I, Cutruzzola F, Antonov A, Agostini M and Melino G. Serine and glycine metabolism in cancer. Trends in biochemical sciences. 2014; 39:191-198.

51. Jochemsen AG. Reactivation of p53 as therapeutic intervention for malignant melanoma. Current opinion in oncology. 2014; 26:114-119.

52. Lezina L, Purmessur N, Antonov AV, Ivanova T, Karpova E, Krishan K, Ivan M, Aksenova V, Tentler D, Garabadgiu AV, Melino G and Barlev NA. miR-16 and miR-26a target checkpoint kinases Wee1 and Chk1 in response to p53 activation by genotoxic stress. Cell death \& disease. 2013; 4:e953.

53. Marouco D, Garabadgiu AV, Melino G and Barlev NA. Lysine-specific modifications of p53: a matter of life and death? Oncotarget. 2013; 4:1556-1571.

54. Blagosklonny MV. P53: An ubiquitous target of anticancer drugs. Int J Cancer. 2002; 98:161-166.

55. Selivanova G and Wiman KG. Reactivation of mutant $\mathrm{p} 53$ : molecular mechanisms and therapeutic potential. Oncogene. 2007; 26:2243-2254.

56. Wang H, Chu W, Das SK, Ren Q, Hasstedt SJ and Elbein SC. Liver pyruvate kinase polymorphisms are associated with type 2 diabetes in northern European Caucasians. Diabetes. 2002; 51:2861-2865.

57. Kraljevic S, Stambrook PJ and Pavelic K. Accelerating drug discovery. EMBO reports. 2004; 5:837-842.

58. Grigoriev I, zu Castell W, Tsvetkov $\mathrm{P}$ and Antonov AV. AERS spider: an online interactive tool to mine statistical associations in Adverse Event Reporting System. Pharmacoepidemiology and drug safety. 2014; 23:795-801.

59. Eichler HG, Bloechl-Daum B, Abadie E, Barnett D, Konig F and Pearson S. Relative efficacy of drugs: an emerging issue between regulatory agencies and third-party payers. Nature reviews Drug discovery. 2010; 9:277-291.

60. Amelio I, Gostev M, Knight RA, Willis AE, Melino G and Antonov AV. DRUGSURV: a resource for repositioning of approved and experimental drugs in oncology based on patient survival information. Cell death \& disease. 2014; 5:e1051.

61. Antonov AV, Krestyaninova M, Knight RA, Rodchenkov I, Melino G and Barlev NA. PPISURV: a novel bioinformatics tool for uncovering the hidden role of specific genes in cancer survival outcome. Oncogene. 2014; 33:1621-1628.

62. Knight RA, Gostev M, Ilisavskii S, Willis AE, Melino G and Antonov AV. Large scale integration of drug-target information reveals poly-pharmacological drug action mechanisms in tumor cell line growth inhibition assays. Oncotarget. 2014; 5:659-666.

63. Peters JU. Polypharmacology - foe or friend? Journal of medicinal chemistry. 2013; 56:8955-8971.

64. Landré V, Amelio I, Barlev NA, Knight RA, Lisitsa A, Melino G and Antonov AV. Perspective on Multi-Target Antiplatelet Therapies: High Content Phenotypic Screening as an Unbiased Source of Novel Polypharmacological Strategies. Mini-Rev Med Chem. 2015; 15:622-9.

65. Blagosklonny MV. Matching targets for selective cancer 
therapy. Drug discovery today. 2003; 8:1104-1107.

66. Blagosklonny MV. Overcoming limitations of natural anticancer drugs by combining with artificial agents. Trends in pharmacological sciences. 2005; 26:77-81. 